\title{
Commitment to Exercise: The Influence of Personal, Athletic, and Psychological Characteristics
}

\author{
Compromiso con el ejercicio: la influencia de \\ características personales, atléticas y psicológicas
}

Recibido: julio 10 de 2011 | Revisado: junio 12 de 2012 | Aceptado: julio 21 de 2012

\author{
RUI GOMES* \\ TATIANA CAPELÃO \\ University of Minho, Braga, Portugal
}

doi:10.11144/Javeriana.UPSY12-2.ceip

Para citar este artículo: Gomes, R. \& Capelão, T. (2013). Commitment to exercise: The Influence of personal, athletic, and psychological characteristics. Universitas Psychologica, 12(2), 505-515.

* Universidade do Minho, Escola de Psicologia. Campus de Gualtar 4710-057 Braga, Portugal. Telf. +253.604.232. Fax: +253.604 .224 . E-mail: rgomes@psi.uminho.pt

\begin{abstract}
A B S T R A C T
This study analyzes the influence of personal, psychological and athletic characteristics of participants regarding the commitment to exercise practice. The study included 153 participants divided in the adherer group $(N=102)$ and the dropout group $(N=51)$. Personal and athletic information, exercise attitudes, perceived behavioral control, intended exercise frequency, goal orientations, behavioral regulation, and expected exercising outcomes were evaluated. The results showed that the participants in the adherer group expressed a greater desire to maintain or increase their weight, they had more experience exercising, had greater intrinsic regulation, and assumed more positive and fewer negative exercise expectations. In conclusion, considering the role of personal, athletic, and psychological characteristics seems important in order to prevent abandoning exercise altogether. Key words authors

Dropout, Exercise, Personal and Athletic Factors, Psychological Factors.

Key words plus

Sport Psychology, Quantitative Research, Descriptive Design.
\end{abstract}

\section{RESUMEN}

Este estudio analiza la influencia de características personales, deportivas y psicológicas en la permanencia o abandono del ejercicio físico. Los 153 participantes fueron divididos en dos grupos: adhesión $(N=102)$ y abandono $(N=51)$. Se recogió información sobre características personales y deportivas, actitudes, percepción de control conductual, intención de frecuencia del ejercicio, orientaciones de meta, regulación conductual y expectativas de ejercicio. Se verificó que el grupo de adhesión mostraba mayor deseo de conservar o aumentar el peso, experiencia en la práctica del ejercicio, regulación intrínseca, así como expectativas más positivas hacia el ejercicio físico. En resumen, conocer el papel de las características personales, deportivas y psicológicas en el ejercicio es importante para la prevención del abandono.

Palabras clave autores

Abandono, ejercicio, factores personales y deportivos, factores psicológicos.

Palabras clave descriptores

Psicología del deporte, investigación cuantitativa, diseño descriptivo. 
Several benefits are associated with exercise (American College of Sports Medicine, 2006; Dishman, Washburn \& Heath, 2004). However, the majority of adults are either sedentary or exercise with a frequency duration, and intensity that does not produce significant advantages (Dishman \& Buckworth, 2001; Martinez-Gonzalez et al., 2001). Moreover, research has demonstrated that exercise drop out levels are very significant. For example, there is evidence that almost half of those who start an exercise program quit within the first six months (Dishman, 1991; Marcus et al., 2000). Consequently, it could be said that besides from the difficulties of motivating people to adhere to exercise, there is also another significant challenge associated with convincing people to maintain an exercise routine. It is also interesting to note that we can find a significant number of studies in the literature about the factors that can explain why people do not start exercising and about the factors that contribute to beginning and maintaining an exercise program (Rhodes, Plotnikoff \& Courneya, 2008; Sheeran \& Abraham, 2003). However, less is known about what causes people to stop exercising. In addition, most of the studies written about the maintenance and abandonment of an exercise program are outdated. The majority of these studies are from the 1970s and 1980s (for a review, see Biddle \& Mutrie, 2008).

Considering the gaps in these studies, it is interesting to consider whether there are differences between people who maintain and abandon the practice of exercise. The main goal of this work was to address this dearth of studies by analyzing whether individuals who commit to or leave exercising really differ in terms of personal, athletic, and psychological characteristics.

Regarding personal characteristics, three variables were chosen in this study (gender, body mass index-BMI, and desire of ideal weight) because there are many studies on the impact of these variables on exercise (Nigg, Lippke \& Maddock, 2009; Rhodes, Blanchard \& Blacklock, 2008). Nevertheless, it is less clear whether these variables make a difference in the tendency to abandon exercise. For the same reason, two athletic variables (attraction towards exercise and past exercise behavior) were selected that have previously been researched in the practice of exercise (Armitage, 2005; Mohiyeddini, Pauli \& Bauer, 2009) but less is known about their influence in the tendency to maintain or abandon exercise.

To determine the influence of psychological characteristics, three sets of variables were used. The first set of variables included exercise attitudes, perceived behavioral control, and intended exercise frequency. These are the main constructs of the theory of planned behavior (Ajzen, 1991), which is one of the major predictive models of exercise behavior (Biddle \& Nigg, 2000). The theory of planned behavior suggests that the proximal determinant of behavior is one's intention to engage in that behavior. This intention is, in turn, influenced by three other factors: attitudes, subjective norms, and perceived behavioral control (Ajzen, 1991). Attitudes can be positive or negative, representing overall evaluations regarding a specific behavior. Subjective norms reflect the individual perception of social pressures from significant people (ex: spouse, friends, etc.) to assume or not assume a specific behavior. Perceived behavioral control assesses the individual perception of control in order to assume or not assume a specific behavior. For the purpose of this study, a measure of exercise attitudes and perceived behavioral control was included. The measure of subjective norms was excluded because there is some evidence that attitudes and perceptions of control are more decisive in determining the behavioral intentions than the perceptions of pressure from others (Armitage \& Conner, 2001; Hagger, Chatzisarantis \& Biddle, 2002).

The second set of variables is related to motivation, namely the goal orientations and the behavioral regulation in exercise. Motivation is an important factor in explaining human behavior, and has a positive relation to the practice of exercise (Dishman, 1994). Recently, a growing number of empirical studies have employed the self-determination theory (Deci \& Ryan, 2000) to analyze the exercise behavior (for a review, see Hagger \& Chatzisarantis, 2007). According to this theory, there are different forms of motivation 
that indicate qualitatively distinct ways in which a behavior can be regulated (Markland \& Tobin, 2004). Thus, intrinsic and extrinsic motivations were proposed as important variables of the theory (Deci \& Ryan, 2000). These autonomous (e.g., intrinsic) and controlled (e.g., extrinsic) types of motivational regulation have an impact on the individual's psychological well-being, persistence in the task, and learning. Intrinsic motivation represents the standard indicator of autonomous motivation, meaning that an individual engages in a certain activity due to his own interest and enjoyment. In this case, the individual formulates intrinsic goals that are mainly focused on developing his personal interests, values, and potential. Extrinsic motivation suggests that the individual engages in a certain activity due to some separable consequences. In this case, the individual formulates extrinsic goals that are mainly characterized by having an "outward" orientation. In other words, the individual's pursuit is directed towards external indicators of worth such as wealth, fame, and appealing image (Sebire, Standage $\&$ Vansteenkiste, 2009). Extrinsic motivation can be divided into four specific regulations that vary in their level of self-determination (Deci \& Ryan, 2000). First, external regulation represents the least autonomous form of extrinsic motivation because the individual becomes involved in an activity due to possible extrinsic rewards or coercion from other people. The second type of motivational regulation is the introjected regulation, meaning that people engage in an activity to pursue contingent self-worth and pride, or to avoid feelings of self-guilt or shame, and not for any form of obligation. The third type of motivational regulation is the identified regulation that represents a relatively self-determined type of extrinsic motivation, meaning that the individual becomes involved in an activity because he accepts and identifies the underlying value of a behavior. The fourth type of motivational regulation is integrated regulation, that occurs when identified regulations are fully assimilated into the self and are congruent with one's values and beliefs (Moller, Deci \& Ryan, 2006; Taylor, Ntoumanis, Standage \& Spray, 2010).
These four behaviors are extrinsic because they are assumed in order to achieve an external regulated goal (e.g., to have a healthy lifestyle), as opposed to assume a behavior for the intrinsic satisfaction and enjoyment of the activity (Deci \& Ryan, 2000). Considering these indications, two measures were included in this study. The first variable measured different forms of behavioral regulations in exercise contexts (i.e., The Behavioral Regulation in Exercise Questionnaire, BREQ-2; Markland \& Tobin, 2004). This version of the instrument also includes the amotivation scale that mainly assesses a failure on the individual's part to value exercise. This instrument has been a valid measure to distinguish between individuals at different stages of exercise changes, and is now used in this study in order to evaluate whether individuals who commit to or leave the practice of exercise differ in terms of behavioral regulation.

The second selected measure evaluates goal orientations (i.e., Goal Orientations in Exercise Measure; Petherick \& Markland, 2008) that analyzes the way people define success in exercise. In this case, people can be orientated towards the task, defining success in exercise based on self-referenced criteria (e.g., increasing effort) or can be orientated towards the ego, defining success in exercise based on the adequacy of personal ability and the demonstration of superior competence, compared with other people who exercise (Petherick \& Markland, 2008). There is some evidence that goals can influence participation in physical activity (Harwood, Wilson \& Hardy, 2003; Kilpatrick, Bartholomew \& Riemer, 2003) but less is known about the impact on the maintenance of exercise. Thus, using these two measures, it was possible not only to analyze the behavioral regulation in exercise, but also to analyze the participants' goal orientation.

The third measure included in the study evaluates the positive and negative expectations of exercise. The positive and negative expectations (or the "pros" and "cons" of exercise) have been incorporated into the transtheoretical model referring to the individual's assessment of the benefits and costs of changing a specific behavior (Prochaska, Redding, Harlow, Rossi \& Velicer, 1994). With regard 
to exercise, pros and cons represent valid dimensions to discriminating among different stages of exercise readiness (Jordan, Nigg, Norman, Rossi \& Benisovich, 2002), being now tested in this study to discern how they may have an impact on people who maintain or abandon exercise.

In sum, this study assumed a correlational and cross-sectional nature, analyzing the differences between individuals who commit to or leave an exercise routine, by using three sets of variables that are rooted in major models that explain exercise behavior (i.e., theory of planned behavior, selfdetermination theory, and transtheoretical model). As Courneya (1995) argues, there are advantages to integrating the elements of different theoretical frameworks when explaining the factors that promote exercise behavior.

Two research questions were defined for this study:

i) Do participants who adhere to and abandon the practice of exercise differ in their personal and athletic characteristics?

ii) Do participants who adhere to and abandon the practice of exercise differ in their psychological characteristics?

\section{Method}

\section{Participants}

The study involved 153 participants, divided into two groups: the adherer group $(N=102,66.7 \%)$ and the dropout group $(N=51,33.3 \%)$. The participants were mainly females $(N=111 ; 72.5 \%)$, and the mean age of the adherer group was 32.37 $(S D=12.87)$ and 37.88 for the dropout group $(S D=12.67)$. It should be reported that there were significant differences between the age groups $\left(t_{(151)}=-2.52, p<=0.05\right)$.

\section{Instruments}

Demographic and athletic information. This questionnaire was developed for the current study, it evaluated personal information (e.g., gender, age, weight, height, and desire for an ideal weight) and athletic information (e.g., attraction towards exercise and past exercise behaviors). Self-reported current weight and height measurements were used to determine body mass indices. The desire for an ideal weight was obtained by asking participants if they would like to have a weight higher, lower, or the same as their current weight. Attraction towards exercise was obtained by asking the participants whether they liked to exercise using a Likert scale $(0=$ not at all, $3=$ very $m u c h)$. Self-reported past exercise behavior was obtained by asking the participants how long they had been exercising, offering four periods of time (up to 6 months, more than 6 months to 1 year, more than 1 year to 5 years, and more than 5 years).

Exercise Attitudes (Ajzen, 2002). Attitude toward exercise was measured using a 7-point bipolar adjective scale that consisted of three items used to evaluate the instrumental attitude component (e.g., useful/useless, wise/foolish, beneficial/harmful; Cronbach's $\alpha=0.81$ ) and three items to evaluate the affective attitude component (e.g., enjoyable/ unenjoyable, interesting/boring, relaxing/stressful; $\alpha=0.85)$. The statement that precedes the adjective was "For me, practicing regular exercise over the next three months will be...".

Perceived Behavioral Control (Ajzen, 2002). Perceived behavioral control was measured by averaging the responses to the following three items: "I am confident that I will be able to perform regular physical activity in the next 4 weeks/2 months/3 months". Answers were given using a Likert format from not at all true for me to completely true for me $(\alpha=0.97)$.

Intended Exercise Frequency. Intended exercise frequency was measured by one item. The participants were asked to rate their perception of exercise frequency per week. Answers were given rating the frequency of exercise from once a week to every day.

Goal Orientations in Exercise Measure (Petherick \& Markland, 2008; Portuguese adaptation by Gomes, 2009). This instrument evaluated the goal orientations of the people who were exercising. These goal orientations represent individual differences with regard to how people construe their per- 
ception of success related to their physical activity. Two major goal perspectives were evaluated: i) taskoriented goals (5 items; $\alpha=0.8$, e.g., "I exercise to the best of my ability"); and ii) ego-oriented goals (5 items; $\alpha=0.86$, e.g., "Other people who exercise don't do as well as me"). The responses were scored on a 5-point Likert scale ranging from 1 (Strongly disagree) to 5 (Strongly agree).

The Behavioral Regulation in Exercise Questionnaire-2 (Markland \& Tobin, 2004; Portuguese adaptation by Palmeira, Teixeira, Silva \& Markland, 2007). This instrument evaluates behavioral regulation in exercise contexts, assessing five dimensions: i) external regulation ( 4 items; $\alpha=0.78$, e.g., "I exercise because other people say I should"); ii) introjected regulation ( 3 items; $\alpha=0.61$, e.g., "I feel guilty when I don't exercise"); iii) identified regulation ( 4 items; $\alpha=0.53$, e.g., "I value the benefits of exercise"); iv) intrinsic regulation ( 4 items; $\alpha=0.75$, e.g., "I exercise because it's fun"); and v) amotivation (4 items; $\alpha=0.75$, e.g., "I don't see why I should have to exercise"). Alpha coefficients revealed significant problems in the identified regulation scale, which resulted in its removal from the following analyses. Introjected regulation was maintained in the analyses due to the fact that there were only three items and an alpha value above 0.6 (Cortina, 1993). Responses were scored on a 5-point scale ranging from 0 (not true for me) to 4 (very true for me).

Expectations of Exercise (Cruz \& Gomes, 2006). This instrument evaluates the positive and negative expectations of exercise that participants perceived by doing physical exercise, the number of times they had previously described in the Intended Exercise Frequency questionnaire. Positive expectations included three items $(\alpha=0.81$, e.g., coping better with daily hassles); and the negative expectations also included three items ( $\alpha=0.82$, e.g., not having enough time for other things). Responses were scored on a 7-point scale ranging from 1 (does not apply me) to 7 (applies to me).

\section{Procedure}

The current study followed the ethical procedures outlined in the Declaration of Helsinki. The col- lection of data involved the following steps: i) a meeting occurred with the manager of a fitness center in order to explain the research goals and the data collection procedures; ii) after approval from the fitness center manager was received, members of the fitness center were invited to participate in the study and were assured that their data would remain anonymous and confidential. Participants were specifically asked to authorize the researchers to have access to their exercise frequency in the following months. Only participants who agreed to these conditions were included in the study, and all of them provided written informed consent before participating.

Data collection occurred mainly at the fitness center before or after an exercise session, being defined a specific room to fill in the evaluation protocol. In some cases, participants asked to fill in the protocol at home and, in these cases, it was defined two occasions to collect the questionnaires (the first one to distribute questionnaires, which were taken home to complete, and the second one to collect the questionnaires). Two hundred and eleven questionnaires were distributed, and 153 were collected and were considered valid (the return rate was $72.5 \%$ ).

\section{Group's Definition}

Participants were selected for each group based on their objective exercise and frequency in the fitness center. Accordingly, in the first time point of evaluation, participants fulfilled the evaluation protocol that informed them about the researchers' access to their rates of exercise frequency. They answered the measures thinking about their exercise practice in that specific fitness center, being excluded other forms of exercice that participants could do outside the fitness center. In this way, it was possible to have a better estimative of the impact of the dimensions evaluated in this study regarding the exercise practice in that specific place.

Then, six months later (second time point of evaluation) we returned to the fitness center and collected the exercise frequency of the participants in order to define the two groups. This frequency of exercise was based on objetive registrations of 
training sessions that each participant has assumed during the six months. The adherer group included the participants that maintained the exercise practice since their first evaluation with a minimum of three exercise sessions per week (American College of Sports Medicine, 2009) and the dropout group included the participants that had no exercise record in the last two months. This period of two months clearly exceeds the one adopted by Ward and Morgan (1984) (e.g., individuals who miss more than two consecutive weeks of exercise practice). In sum, the analysis that follows were done taking into consideration the evaluation protocol collected on the first time point of evaluation and the rates of exercise frequency collected on the second time point of evaluation.

\section{Results}

The Association Between the Practice of Exercising and Personal and Athletic Variables

We tested the association between the exercise practice groups (i.e., the adherer group and the dropout group) and the participants' gender, BMI, desire for an ideal weight, attraction towards exercise, and past exercising behavior. Both chi square tests and logistic regression analyses were conducted to test these associations.

Starting with the personal variables, there were no statistically significant differences between groups for gender $\left(\chi_{(1)}^{2}=2.36\right.$, n.s. $)$ and $\operatorname{BMI}\left(\chi_{(1)}^{2}=0.91\right.$, n.s. $)$. In this second case, two groups were defined: the one with a normal weight $(N=116,75.8 \%)$ and the one considered to be overweight $(N=30,19.6 \%)$. However, significant differences were found in the desire for an ideal weight $\left(\chi_{(1)}^{2}=6.73, p<0.01\right)$. In this case, two groups were constituted: the participants that would like to lose weight ( $N=71,47 \%)$, and the participants that would like to maintain their weight, plus the participants that would like to increase their weight $(N=80,53 \%)$.

In fact, in the adherer group, the majority of the participants expressed a desire to maintain or even increase their weight $(N=61,60.4 \%)$ whereas in the dropout group, the majority of the participants expressed a desire to reduce their weight $(N=31$, $60.2 \%$ ). Also, the participants in the dropout group were 2.49 times more likely than the participants in the adherer group to desire weighing less (Wald $\chi^{2}=6.58, p<0.05 ;$ OR $=2.49$, CI 1.24-4.99).

Regarding the athletic variables, there were no statistically significant differences between the groups regarding their attraction to exercise $\left(\chi_{(1)}^{2}=1.28\right.$, n.s.). In this case, the groups were divided between the participants that had a lower to moderate attraction to exercise $(N=79$, $53.7 \%)$ and the ones with a higher attraction to exercise $(N=68,46.3 \%)$. For previous exercising behavior, significant differences were found $\left(\chi_{(1)}^{2}=5.43, p<0.05\right)$. In this case, two groups were defined: the participants that were exercising up to one year $(\mathrm{N}=24,15.8 \%)$ and the participants that had been exercising for more than one year $(N=128,84.2 \%)$. In fact, in the adherer group, the majority of the participants had been exercising for more than one year $(N=90,70.3 \%)$, whereas in the dropout group the majority of the participants had been exercising for less than one year $(N=13,54.2 \%)$. Despite these differences, logistic regression analyses did not point out differences between groups (Wald $\chi^{2}=0.00$, .n.s.; $\mathrm{OR}=0.98$, CI 0.48-2.00).

\section{Exercise Practice and Psychological Differences}

This analysis tested differences between exercise practice groups (i.e., adherer and dropout groups) on the evaluated psychological dimensions (e.g., exercise attitudes, perceived behavioral control, intended exercise frequency, goal orientations, behavioral regulation in exercise, and exercise expectations). These differences were tested using $t$-tests for the independent samples to the global score of the unidimensional instruments and a multivariate analysis of variance to the subscales of the multidimensional instruments. Before testing the differences, three steps were followed in the data analysis: i) we start by doing an exploratory data analysis in 
order to test the assumptions underlying the parametric tests, and in general all the conditions were met (ex: independence of observations, normal distribution of the results regarding the dependent variable, homogeneity of the matrix of variancecovariance, etc.); ii) when normality could not be assumed, we computed both the parametric and the equivalent non-parametric tests, as advised by FifeSchaw (2006). In all cases, the results from both tests allowed us to draw the same conclusions; and iii) because we observed similar results using both the parametric and the equivalent non-parametric tests, we only present results from the parametric tests because they are more robust and permit us to use multivariate analysis. This also reduces the number of tests conducted and, therefore, the probability of a Type I error.

As a result, no differences were found between the groups regarding exercise attitudes, (Wilks' $\lambda=0.98, F_{(2,138)}=1.27$, n.s., $\left.\eta^{2}=0.02\right)$, perceived behavioral control $\left(t_{(145)}=-1.32\right.$, n.s. $)$, exercise frequency intention $\left(t_{(146)}=-0.14\right.$, n.s.), and goal orientations (Wilks' $\lambda=0.99, F_{(2,148)}=0.99$, n.s., $\left.\eta^{2}=0.01\right)$. In this same way, no differences were found between groups regarding behavioral regulation in exercise (Wilks' $\lambda=0.96, F_{(4,144)}=1.50$, n.s., $\left.\eta^{2}=0.04\right)$. However, both the univariate parametric tests and the non-parametric tests point out marginal significant differences between the groups in the intrinsic regulation scale $\left(\mathrm{F}_{(1,147)}=3.06\right.$, $p<0.01, \eta^{2}=0.02$ ). Higher values in the adherer group were observed when compared with the dropout group.

Finally, significant differences between the groups were found in terms of the exercise expectations (Wilks' $\lambda=0.93, F_{(2,149)}=5.37, p<0.01$, $\left.\eta^{2}=0.07\right)$. Univariate tests revealed that the adherer group assumed more positive exercise expectations $\left(\mathrm{F}_{(1,150)}=5.33, p<=0.05, \eta^{2}=0.03\right)$ and less negative exercise expectations $\left(\mathrm{F}_{(1,150)}=5.76\right.$, $\left.p<=0.05, \eta^{2}=0.04\right)$ than the dropout group.

\section{Discussion}

Comprehending the factors involved in exercise behavior is a very challenging task due to the need of explaining the reasons why some people start exercising while others do not, and why some people maintain exercising while others give up. However, as Armitage (2005) argues, there is a dearth of research on the maintenance of exercise behavior. A possible explanation for this scarcity may be the fact that researchers have considered behavior change to be a process, with maintenance as the last step (Wing, 2000). On the contrary, the maintenance behavior should itself be considered a process that can have different results, such as relapsing and abandoning the initial behavior. Thus, this study assumes that exercise behavior can change, resulting in the maintenance or abandonment of the initial behavior. Also, this study evaluated exercise behavior through the rates of the participants' exercise frequency, dividing the adherer group and the dropout group based on objective measures of exercise behavior. As stated by Armitage (2005), research has been measuring exercise behavior with single-occasion self-reports that could be quite problematic because they are susceptible to memory biases. In this way, this study used objective measures of exercise behavior in order to define the participants' status regarding exercise (maintain or abandon of exercise) using for that the registrations of exercise practice through a period of six months. This need of analyzing the impact of psychological factors on the effective exercise behavior has been reinforced in the literature (Armitage, 2005; Norman \& Conner, 2005), being this aspect important because most studies done so far tend to use the intention of doing exercise or the subjective perception of exercise practice as main indicators of exercise behavior. Taking into consideration these ideas, this study analyzed whether participants' personal, athletic, and psychological characteristics influence their effective exercise behavior.

Starting with the personal and athletic variables, the results reinforced two aspects. First, gender, BMI, and attraction towards exercise did not reveal any significant differences between the adherer group and the dropout group. A scarce amount of findings exists about the importance of these variables on the maintenance or abandonment of exercise. For example, Mohiyeddini et 
al. (2009) provide indications about the fact that males and females did not differ in their routines of training duration and frequency, and it is now also evident that no differences exist in their tendency to maintain or abandon exercise.

Second, in the case of the desire for an ideal weight and the past exercise behavior, significant differences were found. Accordingly, having the desire to maintain the same weight or even want to increase the weight, and have been doing exercising for more than one year were positively associated with maintaining the exercise behavior. In both cases, a scarcity of information exists regarding the impact of these variables on exercise behavior. However, it is interesting to note that the desire for an ideal weight lower than the current one has already been associated with eating disordered behaviors in athletes (Gomes, Martins \& Silva, 2011), being now associated with the dropout of exercise in a non-athletic sample. In the case of previous exercise behavior, Armitage's (2005) earlier suggestion that this variable could represent an index of maintenance in the practice of exercise is confirmed. In addition, it is now evident that previous exercising behavior should also be considered when analyzing the factors that explain the abandonment of exercising.

Regarding the differences between the adherer group and the dropout group in the psychological dimensions, three aspects deserve attention. First, none of the dimensions based on the theory of planned behavior assumed significant differences between the groups. This confirms the idea that the model seems more useful in explaining exercise intentions but not as much in explaining the current (Norman \& Conner, 2005) and future exercise behavior. Second, in the self-determination theory, goal orientations did not reveal any differences between groups, and from the four dimensions considered in the BRESQ-2, only intrinsic regulation assumed marginal significant differences between the groups. In this case, the adherer group assumed a higher autonomous motivation, meaning that exercise was perceived more as a personal activity that promoted fun and enjoyment. This result is important because autonomous motivation in exercise has been referenced as an important predictor of performing moderate-intensity exercise (Standage, Sebire \& Loney, 2008), being now also related with differentiating groups of individuals who maintain or abandon exercise.

Third, the most evident differences were related with the exercise expectations. In fact, the adherer group assumed both more positive and fewer negative exercise expectations than the dropout group. This result supports the interest of the transtheoretical model (Prochaska et al., 1994) in explaining exercise behavior, not only when individuals decide to start an exercise program, but also when they decide to continue or abandon their exercise routine.

This study has some limitations. First, mean ages of the adherer group and the dropout group were different, being the dropout group older than the adherer group. This aspect is important because the engagement in physical activity declines with age (Armstrong \& McManus, 1994). Another potential limitation is that the sample included people doing their exercise in a private fitness center, which is not representative of a random sample of the general population. Besides, it is also possible that some participants included in the dropout group could have assumed other forms of exercise outside the fitness center, and because of that they may not be considered effective dropouts of exercise. However, we should mention that the main goal of this study was to analyze the differences between the adherer and the dropout groups regarding the collected measures, considering for that their rates of exercise frequency in that specific fitness center. Because of that, all the participants were specifically asked to give their answers in the evaluation protocol thinking about that particular place of exercise practice. So they knew that we were not evaluating other forms of exercise outside the fitness center.

In terms of future research, it would be important to design studies in order to collect data in two time points. The first one would be when all the participants were involved in exercise practice (as was the case in this study). In the second time point, the psychological measures would be collected again in the adherer and dropout groups in order to analyze differences across time. Of course, 
this option should be carefully designed due the difficulties of collecting data from the dropout group.

Finally, the results illustrate some important practical implications. When preventing dropout behavior, the personal and athletic characteristics of the people exercising (namely, the aspects related with the desire for an ideal weight and past exercising behavior) as well as some psychological factors should be considered. In this case, increasing the personal significance of exercise by turning this activity into something interesting and fun seems an effective way to promote the maintenance of an exercise program. But most importantly, reducing the obstacles to exercise and increasing the positive exercise expectations could indeed promote the possibility of maintaining an exercise program and reduce the undesirable drop out behavior. As mentioned in the beginning of this paper, it is important to know the factors that influence people to adhere to exercise, but it is also important to know what factors lead to the maintenance of an exercise program.

\section{References}

Ajzen, I. (1991). The theory of planned behavior. Organisational Behavior and Human Decision Processes, 50(2), 179-211.

Ajzen, I. (2002). Construction of a standard questionnaire for the theory of planned behaviour. Retrieved from http://www-unix.oit.umass.edu/ aizen/

American College of Sports Medicine. (2006). ACSM's guidelines for exercise testing and prescription (7th ed.). Baltimore, MD: Lippincott Williams \& Wilkins.

American College of Sports Medicine. (2009). ACSM's guidelines for exercise testing and prescription (8th ed.). Baltimore, MD: Lippincott Williams \& Wilkins.

Armitage, C. J. (2005). Can the theory of planned behavior predict the maintenance of physical activity? Health Psychology, 24(3), 235-245.

Armitage, C. J. \& Conner, M. (2001). Efficacy of the theory of planned behaviour: A meta-analytic review. British Journal of Social Psychology, 40(4), 471-499.
Armstrong, N. \& McManus, A. (1994). Children's fitness and physical activity: A challenge for physical education. British Journal of Physical Education, 25(1), 20-26.

Biddle, S. J. H. \& Mutrie, N. (2008). Psychology of physical activity: Determinants, well-being and interventions ( $\left.2^{\text {nd }} \mathrm{ed}.\right)$. London: Routledge.

Biddle, S. J. H. \& Nigg, C. R. (2000). Theories of exercise behavior. International Journal of Sport Psychology, 31(2), 290-304.

Cortina, J. M. (1993). What is coefficient alpha? An examination of theory and applications. Journal of Applied Psychology, 78(1), 98-104.

Courneya, K. S. (1995). Understanding readiness for regular physical activity in older individuals: An application of the theory of planned behavior. Health Psychology, 14(1), 80-87.

Cruz, J. F. \& Gomes, A. R. (2006). Expectativas de Resultado do Exercício-Benefícios e Custos (ERE-BC)

[Expectations of exercise]. Relatório técnico não publicado. Braga: Universidade do Minho.

Deci, E. L. \& Ryan, R. M. (2000). The "what" and "why" of goal pursuits: Human needs and the selfdetermination of behavior. Psychological Inquiry, 11(4), 227-268.

Dishman, R. K. (1991). Increasing and maintaining exercise and physical activity. Behaviour Therapy, 22(3), 345-378.

Dishman, R. K. (1994). Advances in exercise adherence. Champaign, IL: Human Kinetics.

Dishman, R. K. \& Buckworth, J. (2001). Exercise psychology. Champaign, IL: Human Kinetics.

Dishman, R. K., Washburn, R. A. \& Heath, G. W. (2004). Physical activity epidemiology. Champaign, IL: Human Kinetics.

Fife-Schaw, C. (2006). Levels of measurement. In G. M. Breakwell, S. Hammond, C. Fife-Schaw \& J. A. Smith (Eds.), Research methods in psychology (3rd ed., pp. 50-63). London: Sage.

Gomes, A. R. (2009). Questionário de Orientação Motivacional para o Exercício Físico (QOMEF) [Goal Orientation in Exercise Measure]. Relatório técnico não publicado. Braga: Universidade do Minho.

Gomes, A. R., Martins, C. \& Silva, L. (2011). Eating disordered behaviours in Portuguese athletes: The influence of personal, sport, and psychologi- 
cal variables. European Eating Disorders Review, 19(3), 190-200.

Hagger, M. S. \& Chatzisarantis, N. L. D. (2007). Advances in self-determination theory research in sport and exercise. Psychology of Sport and Exercise, 8(5), 597-599.

Hagger, M. S., Chatzisarantis, N. L. D. \& Biddle, S. J.H. (2002). A meta-analytic review of the theories of reasoned action and planned behavior in physical activity: Predictive validity and the contribution of additional variables. Journal of Sport and Exercise Psychology, 24(1), 3-32.

Harwood, C., Wilson, K. \& Hardy, L. (2003). Achievement goals in sport: Working towards an alternative model. Journal of Sports Sciences, 21(4), 349-350.

Jordan, P. J., Nigg, C. R., Norman, G. J., Rossi, J. S. \& Benisovich, S. V. (2002). Does the transtheoretical model need an attitude adjustment? Integrating attitude with decisional balance as predictors of stage of change for exercise. Psychology of Sport and Exercise, 3(1), 65-83.

Kilpatrick, M., Bartholomew, J. \& Riemer, H. (2003). The measurement of goal orientations in exercise. Journal of Sport Behavior, 26(2), 121-136.

Marcus, B. H., Dubbert, P. M., Forsyth, L. H., McKenzie, T. L., Stone, E. J., Dunn, A. L., et al. (2000). Physical activity behavior change: Issues in adoption and maintenance. Health Psychology, 19(1), 32-41.

Markland, D. \& Tobin, V. (2004). A modification of the behavioral regulation in exercise questionnaire to include an assessment of amotivation. Journal of Sport and Exercise Psychology, 26(2), 191-196.

Martinez-Gonzalez, M. A., Varo, J. J., Santos, J. L., De Irala, J., Gibney, M., Kearney, J., et al. (2001). Prevalence of physical activity during leisure time in the European Union. Medicine $\mathbb{E}$ Science in Sports E Exercise, 33(8), 1142-1146.

Mohiyeddini, C., Pauli, P. \& Bauer, B. (2009). The role of emotion in bridging the intention-behaviour gap: The case of sports participation. Psychology of Sport and Exercise, 10(2), 226-234.

Moller, A. C., Deci, E. L. \& Ryan, R. M. (2006). Choice and ego-depletion: The moderating role of autonomy. Personality and Social Psychology Bulletin, 32(8), 1024-1036.
Nigg, C. R., Lippke, S. \& Maddock, J. E. (2009). Factorial invariance of the theory of planned behavior applied to physical activity across gender, age, and ethnic groups. Psychology of Sport and Exercise, 10(2), 219-225.

Norman, P. \& Conner, M. (2005). The theory of planned behavior and exercise: Evidence for the mediating and moderating roles of planning on intentionbehavior relationships. Journal of Sport $\mathbb{E}$ Exercise Psychology, 27(4), 488-504.

Palmeira, A., Teixeira, P., Silva, M. \& Markland, D. (2007, September). Confirmatory factor analysis of the behavioural regulation in exercise questionnaire - Portuguese version. Paper presented at the 12 th European Congress of Sport Psychology, Halkidiki, Greece.

Petherick, C. \& Markland. D. (2008). The development of a goal orientation in exercise measure (GOEM). Measurement in Physical Education $\mathbb{E}$ Exercise Science, 12, 55-71.

Prochaska, J. O., Redding, C. A., Harlow, L. L., Rossi, J. S. \& Velicer, W. F. (1994). The transtheoretical model of change and HIV prevention: A review. Health Education Quarterly, 21(4), 471-486.

Rhodes, R. E., Blanchard, C. M. \& Blacklock, R. E. (2008). Do physical activity beliefs differ by age and gender? Journal of Sports and Exercise Psychology, 30(3), 412-423.

Rhodes, R. E., Plotnikoff, R. C. \& Courneya, K. S. (2008). Predicting the physical activity intentionbehavior profiles of adopters and maintainers using three social cognition models. Annals of Behavioral Medicine, 36(3), 244-252.

Sebire, S. J., Standage, M. \& Vansteenkiste, M. (2009). Examining intrinsic versus extrinsic exercise goals: Cognitive, affective, and behavioral outcomes. Journal of Sport and Exercise Psychology, 31(2), 189-210.

Sheeran, P. \& Abraham C. (2003). Mediator of moderators: Temporal stability of intention and the intention-behavior relationship. Personality Social Psychological Bulletin, 29(2), 205-215.

Standage, M., Sebire, S. J. \& Loney, T. (2008). Does exercise motivation predict engagement in objectively assessed bouts of moderate-intensity exercise? 
A self-determination theory perspective. Journal of Sport and Exercise Psychology, 30(4), 337-352.

Taylor, I. M., Ntoumanis, N., Standage, M. \& Spray, C. M. (2010). Motivational predictors of physical education students' effort, exercise intentions, and leisure-time physical activity: A multilevel linear growth analysis. Journal of Sport and Exercise Psychology, 32(1), 99-120.
Ward, A. \& Morgan, W. (1984). Adhrence patterns of healthy men and women enrolled in an adult exercise program. Journal of Cardiac Rehabilitation, 4(4), $143-152$.

Wing, R. R. (2000). Cross-cutting themes in maintenance of behavior change. Health Psychology, 19(Suppl. 1), 84-88. 
